\title{
Pressure Effect on Superconducting Critical Temperature According to String Model
}

\author{
Ibrahim Adam Ibrahim Hammad ${ }^{2}$, Mubarak Dirar ${ }^{1}$, Nadia Omar Alatta ${ }^{2}$, Rasha Abd Alhai Taha ${ }^{3}$, \\ Kh. M. Haroun ${ }^{2}$, Rawia A. Elgani ${ }^{3}$ \\ ${ }^{1}$ Department of Physics, Faculty of Science, International University of Africa, Khartoum, Sudan \\ ${ }^{2}$ Department of Physics, Faculty of Education, Alzaiem Alazhari University, Omdurman, Sudan \\ ${ }^{3}$ Department of Physics, Faculty of Science, Sudan University of Science \& Technology, Khartoum, Sudan
}

Email address:

kshiky1986@gmail.com (I. A. I. Hammad)

\section{To cite this article:}

Ibrahim Adam Ibrahim Hammad, Mubarak Dirar, Nadia Omar Alatta, Rasha Abd Alhai Taha, Kh. M. Haroun' Rawia A. Elgani. Pressure Effect on Superconducting Critical Temperature According to String Model. International Journal of Fluid Mechanics \& Thermal Sciences. Vol. 3, No. 6, 2017, pp. 70-74. doi: 10.11648/j.ijfmts.20170306.12

Received: March 2, 2017; Accepted: March 31, 2017; Published: November 28, 2017

\begin{abstract}
Superconductivityis generally regarded as one of the most striking and widely used physical phenomenon. Physicists in response have shown sheer interest in scrutinizing superconductivity and constructing theoretical models to explain it. The majorityof models derived in this regard neglected some aspects of superconductivity. The link between critical temperature and pressure remains a highly neglected and potentially representing a research gap in this area. Thus, this motivates the researchers to construct a new model on the relation between pressure and superconductors critical temperature using a string model. The study mainly aims to construct theoretical model based on string model in attempt to understand the effect of pressure on critical temperature and superconducting resistance. The results of study reveal that using plasma equation for mechanical and thermal pressure the frequency is obtained. It also finds that treating electrons as string the energy is found in terms temperature and pressure. Further, when the superconducting resistance vanishes the corresponding critical temperature was found. Furthermore, the increasing mechanical pressure increases the critical temperature.
\end{abstract}

Keywords: Plasma Equation, Superconductivity, Resistivity, Critical Temperature, Pressure

\section{Introduction}

Superconductivity (Sc henceforth) was first discovered in 1911 by KamerlinghOnnes. Onnes discovered that the element of mercury exhibited zero resistance at critical temperature $\left(\mathrm{T}_{\mathrm{c}}=4.1 \mathrm{~K}\right)[1,2]$, the conditions in which a superconductor retains its superconducting properties is defined by its critical values. These critical values are critical temperature $T_{c}$, the critical magnetic field $B_{c}$ and the critical current density $\mathrm{J}_{\mathrm{c}}$ and depend on the superconducting material [3]. At critical temperature the spacemen undergoes a phase transition from a state normal electrical resistivity to superconducting state when the resistance vanishes abruptly below the $\mathrm{T}_{\mathrm{c}}$ [4]. Another significant discovery was made byMeissner and Ochsenfeldin 1933 when they realized that the magnetic field is expelled out of the body of the superconductor. Meissner and Ochsenfeld found that the magnetic field penetrates the material at a small distance, with an order (30-60) $\mathrm{nm}$ in metal superconductors. They called it London's penetration depth [5]. Superconductors divide into two classes according to behavior in a magnetic field. Allpure samples of superconducting elements, except $\mathrm{Nb}$, exhibit type-1 behaviour and their superconductivity destroyed by a modest applied magnetic field $\mathrm{B}_{\mathrm{c}}$, known as the critical field. The behavior of type- 1 and superconductors, at a given temperature $\mathrm{T}$ and in a uniform external magnetic field $\mathrm{H}$, can be described as follows: if $\mathrm{H}$ is smaller than a critical value $\boldsymbol{H}_{\boldsymbol{c}}(\boldsymbol{T})$, the superconductor completely expels the magnetic flux from its interior (Meissner effect); as the external field is increased above the critical value $\boldsymbol{H}_{\boldsymbol{c}}(\boldsymbol{T})$, the entre specimen reverts from the superconducting to the normal state [6]. Although $\mathrm{Nb}$ is the only element that is type- 2 in is pure state, other elements generally become type- 2 when the electron mean free path is reduced sufficiently by alloying [7]. In type-2 superconductors, the transition to a normal state is quite 
gradual. Where superconductivity is only partially destroyed for $\boldsymbol{H}_{\boldsymbol{C} 1} \leq \boldsymbol{H} \leq \boldsymbol{H}_{\boldsymbol{C} 2}$. The regionbetween $\boldsymbol{H}_{\boldsymbol{C} 1}$ and $\boldsymbol{H}_{\boldsymbol{C} 2}$ is known as intermediate state as it contains partially both normal and the superconducting states. $\boldsymbol{H}_{\boldsymbol{C} 1}$ is called the lower critical field, whereas $\boldsymbol{H}_{\boldsymbol{C} 2}$ is known as upper critical field. At $\boldsymbol{H}_{\boldsymbol{C} 1}$ the field begins to penetrate the sample, and the penetration increases until $\boldsymbol{H}_{\boldsymbol{C} 2}$ is reached. At $\boldsymbol{H}_{\boldsymbol{C} 2}$, the magnetization vanishes and the sample reaches the normal. Type-2 superconductors exhibit imperfect diamagnetism [8]. Type-2 superconductors are known in which $\boldsymbol{H}_{\boldsymbol{C} 2}$ is large as $2.8 \times 10^{7} \boldsymbol{A} / \boldsymbol{m}$ at absolute zero. Such materials are used now for practical superconducting magnet coils, and are anticipated for use in the generation and distribution of electrical power [9].

In 1957 Bardeen, Cooper, and Schrieffer (BCS) proposed a microscopic theory. This marked the firstendeavors in the theoretical understanding of the nature of Sc. The present day theoretical state owes much to this development. They showed that bound electron pairs termed as Cooper pairs carry the supercurrent. There is also an energy gap between the normal and superconducting state [10]. The BCS theory injected two powerful ideas into the collective consciousness: pairing and spontaneous symmetry breaking. Pairing was an essentially new idea, introduced by Cooper and brought to fruition by BCS. The symmetry breaking aspect was mostly implicit in the original BCS work, and in earlier of Fritz London and Landau-Ginzburg; but the depth and success of the BCS theory seized the imagination of the theoretical physics community, and catalyzed and intellectual ferment [11]. The development of Sc lead to discovery of new material of high temperature superconductors (HTSCs), with their superconducting $\mathrm{T}_{\mathrm{c}}$ exceeding $23^{\circ} \mathrm{K}$, a superconductor usually referred to a HTSC if the $\mathrm{T}_{\mathrm{c}}$ exceeds $90^{\circ} \boldsymbol{K}[12,13]$. The observation of HTSCs in complex layered copper oxides (cuprates) by Bednorz and Muller in 1986 should undoubtedly be rated as one of the greatest experimental discoveries of the last century, whereas identifying and understanding the microscopic origin of high-temperature superconductivity stands as one of the greatest theoretical challenges of this century, they discovered the onset of possible superconductivity at exceptionally high temperatures in a black ceramic material comprising four elements: lanthanum, barium, copper and oxygen. Within the next decade many more complex cuprates were synthesized including the mercury cuprate compounds which, to date, have the highest confirmed critical temperature for a superconducting transition, some $\boldsymbol{T}_{\boldsymbol{c}}=135^{\circ} \boldsymbol{K}$ at room pressure and approximately $160^{\circ} \mathrm{K}$ under high applied pressure. The new phenomenon initiated by Bednorz and Muller broke all constraints on the maximum $\boldsymbol{T}_{\boldsymbol{c}}$ predicted by the conventional theory of low-temperature superconducting metals and their alloys. These discoveries could undoubtedly result in large-scale commercial applications for cheap and efficient electricity production, provided long lengths of superconducting wires operating above the liquid nitrogen temperature $\left(80^{\circ} \boldsymbol{K}\right)$ can be routinely manufactured [14]. Pressure was very effective in inducing $\mathrm{Sc}$ in elements and compounds that are not superconducting at ambient pressure, some of these elements such as $\mathrm{Si}, \mathrm{Ge}$ and cuprate are shown to be effected by applying pressure, by application of pressure on these superconducting cuprates increases $\mathrm{T}_{\mathrm{c}}$ for underdoped and optimally doped compounds. For example $\mathrm{T}_{\mathrm{c}}$ of $\boldsymbol{H g B a} \boldsymbol{C a}_{2} \boldsymbol{C u}_{3} \boldsymbol{O}_{8+\delta}\left(135^{\circ} \boldsymbol{K}\right.$ at ambient pressure), which is the highest found among any superconductor, increases up to $164^{\circ} \mathrm{K}$ at $30 \mathrm{GPa}$. It is generally considered that application of pressure promotes transfer of charge from reservoir block to superconducting block. In under doped superconductors, the pressure raises $T_{c}$ by doping while a decrease of $T_{c}$ is observed in overdoped cuprates. In mercury cooperates such as $\boldsymbol{H g B a} \boldsymbol{a}_{2} \boldsymbol{C a}_{2} \boldsymbol{C u}_{3} \boldsymbol{O}_{8+\boldsymbol{\delta}}$ even overdoped samples show an increase of $\mathrm{T}_{c}$ under pressure because intrinsic effects are supposed to be more efficient than charge transfer. A possible explanation is shown that under the action of the pressure, the reservoir blocks shrink more than the superconducting blocks, so that $T_{c}$ increases due to an enhanced proximity effect. In this respect, when the pressure increase the $T_{c}$ increases $[15,16,17,18]$. A plethora of studiesinvestigated the effect of pressure on the $T_{c}$ using an experimental approach. One of these studies dealt withthe pressuredependence of the superconducting critical temperature of the $\mathrm{Tl}_{0.5} \mathrm{~Pb}_{0.5} \mathrm{Sr}_{2} \mathrm{Ca}_{1-\mathrm{x}} \mathrm{Y}_{\mathrm{x}} \mathrm{Cu}_{2} \mathrm{O}_{7}$ system. The results of this studyindicated that thepressure is proportional with $T_{c}$ [19]. Another study examined the effect of pressure on the properties of molybdenum sulfides comparing the superconducting and the normal states. This paper found that when the pressure increases the $T_{c}$ decreases [20].

\section{Pressure Effect on Superconducting Critical Temperature According to String Model}

Plasma equation describes ionized particles in a gaseous or liquid form. This equation describes the electron easy motion. The electrons here behaveas ionized particles inside matter.

The plasma medium has two types of pressure; thermal pressure and mechanical pressure $\left(\mathrm{p}_{\mathrm{m}}\right)$ per particle [21].

Thermal pressure is given by

$$
\mathrm{P}=\mathrm{n} \gamma \mathrm{kT}
$$

Where, $\mathrm{n}, \gamma, \mathrm{k}$ and $\mathrm{T}$; are the number of particles, friction coefficient, Boltzmann constant and temperature respectively. By introducing the two types of pressure, one finds the equation of motion of plasma particles to be

$$
\boldsymbol{m n} \frac{d v}{d x} \frac{d x}{d t}=-\frac{\partial p}{\partial x}+\frac{\partial n p_{m}}{\partial x}+\frac{\partial n V_{o}}{\partial x}
$$

Where, $V_{o}$ is the crystal field.

Thus 


$$
\frac{n}{2} \boldsymbol{m} v^{2}=-n \gamma k T+n p_{m}+V_{o} n
$$

For harmonic oscillator string particles the displacement is given by

$$
x=x_{o} e^{i w t}, v=i w x
$$

Thus inserting equation (4) in (3) yields

$$
-\frac{n m \omega^{2} x^{2}}{2}=-n \gamma k T+n p_{m}+V_{o} n
$$

The positive sign of $\mathrm{p}_{\mathrm{m}}$ is due to the fact that the pressure is exerted on the system.

Then, from equation (5) one finds the frequency to be

$$
\omega=\frac{1}{x} \sqrt{\frac{2}{m}} \sqrt{\gamma k T-V_{o}-p_{m}}
$$

Consider the electron as string harmonic oscillator, thus, depends on the quantum mechanicsthe energy of harmonic oscillator is given by

$$
\boldsymbol{E}=\hbar \omega
$$

But for harmonic oscillator from $\boldsymbol{T}=\frac{1}{2} \boldsymbol{m} \boldsymbol{v}^{2}=\frac{-\boldsymbol{m} \boldsymbol{w}^{2} \boldsymbol{x}^{2}}{2}=\boldsymbol{V}$ i.e $\mathrm{T}=\mathrm{V}$.

Where, $\mathrm{T}$ is the kinetic energy and $\mathrm{V}$ is the potential energy.

Hence the total energy of harmonic oscillator is

$$
\begin{gathered}
\boldsymbol{E}=\hbar \omega=\boldsymbol{T}+\boldsymbol{V}=2 \boldsymbol{V} \\
\boldsymbol{V}=\boldsymbol{e} \boldsymbol{V}_{\boldsymbol{e}}
\end{gathered}
$$

Where, e and $V_{e}$ are the electronic potential and electron charge respectively.

According to classical laws the resistance is given by

$$
R=\frac{V_{e}}{I}=\frac{V}{e I}=\frac{E}{2 e I}=\frac{\hbar \omega}{2 e I}
$$

Sub equation (6) in (10) yields

$$
R=\frac{\hbar}{2 e I x} \sqrt{\frac{2}{m}} \sqrt{\gamma k T-V_{o}-p_{m}}
$$

Splitting $\mathrm{R}$ to real part $\mathrm{R}_{\mathrm{s}}$ and imaginary part $\mathrm{R}_{\mathrm{i}}$

$$
R=R_{r}+i R_{i}
$$

The $\mathrm{R}_{\mathrm{s}}=\mathrm{R}_{\mathrm{r}}$

When

$$
\begin{gathered}
\gamma \boldsymbol{k} T<\boldsymbol{p}_{\boldsymbol{m}}-\boldsymbol{V}_{o} \\
T<\frac{\boldsymbol{p}_{\boldsymbol{m}}-\boldsymbol{V}_{o}}{\gamma \boldsymbol{k}}
\end{gathered}
$$

Then

$$
T_{c}=\frac{p_{m}-V_{o}}{\gamma k}
$$

$\mathrm{R}=$ imaginary $=i \mathrm{R}_{\mathrm{i}}, \mathrm{R}_{\mathrm{r}}=0, \mathrm{R}_{\mathrm{s}}=0$

Eq. (15) shows that when the pressure increases the $T_{c}$ is increases.

Driving both sides of Eq. (3) yields

$$
d\left(\frac{1}{2} n m v^{2}\right)=-d n \gamma k T+d n p_{m}+d V_{o} n
$$

Integrating both side yields

$$
\frac{1}{2} n \boldsymbol{m} v^{2}=-n \gamma k T+n p_{m}+V_{o} n+C_{o}
$$

Or

$$
\begin{gathered}
\boldsymbol{n}\left[\frac{1}{2} \boldsymbol{m} \boldsymbol{v}^{2}+\gamma \boldsymbol{k} T-\boldsymbol{p}_{\boldsymbol{m}}-V_{o}\right]=\boldsymbol{C}_{o}=\mathrm{cons} \tan \boldsymbol{t} \\
E=\left[\frac{1}{2} \boldsymbol{m} \boldsymbol{v}^{2}+\gamma \boldsymbol{k} T-p_{m}-V_{o}\right]
\end{gathered}
$$

For harmonic oscillator Eq. (7) and Eq. (19) one finds

$$
\begin{gathered}
\boldsymbol{T}=\frac{1}{2} \boldsymbol{m} \boldsymbol{v}^{2}=\boldsymbol{V} \\
\boldsymbol{E}=2 \boldsymbol{V}+\gamma \boldsymbol{k} \boldsymbol{T}-\boldsymbol{p}_{\boldsymbol{m}}-\boldsymbol{V}_{o}
\end{gathered}
$$

Or

$$
\begin{aligned}
& \hbar \omega=E=2 \boldsymbol{V}+\gamma k T-p_{m}-V_{o} \\
& R=\frac{<H>}{e I}=\frac{\gamma k T-p_{m}+2 V-V_{o}}{e I}
\end{aligned}
$$

The resistance $R$ can split into two parts, real part $R_{+}$and imaginary part R. [22].

$$
\boldsymbol{R}=\boldsymbol{R}_{+}+\boldsymbol{R}_{-}
$$

Hence

$$
\mathrm{Rs}=\mathrm{R}+, \mathrm{R}=\mathrm{R}-
$$

When

$$
\begin{gathered}
\gamma \boldsymbol{k} \boldsymbol{T}-\boldsymbol{p}_{\boldsymbol{m}}+2 \boldsymbol{V}-\boldsymbol{V}_{o}<0 \\
\boldsymbol{T}<\frac{\boldsymbol{p}_{\boldsymbol{m}}-2 \boldsymbol{V}+\boldsymbol{V}_{o}}{\gamma \boldsymbol{k}}
\end{gathered}
$$

The Sc resistance vanishes as indicated in equations (23), (24) and (26).

Thus critical temperature is given by 


$$
T_{c}=\frac{p_{m}-2 \boldsymbol{V}+V_{o}}{\gamma k}
$$

Consider the definition of $\mathrm{R}$ in terms of potential $\mathrm{V}$, by considering the total energy $\mathrm{E}$ to be of the harmonic oscillator then

$$
\begin{gathered}
E=2 V=\gamma k T-p_{m} \\
V=\frac{\gamma k T-p_{m}}{2} \\
R=\frac{V}{e I}=\frac{\gamma k T-p_{m}}{2 e I}=R_{+}+R_{-}
\end{gathered}
$$

Then, $\mathrm{R}=\mathrm{R}$.

When

$$
\gamma k T-p_{m}<0
$$

Or

$$
T<\frac{p_{m}}{\gamma k}
$$

Thus the critical temperature be

$$
T_{c}=\frac{p_{m}}{\gamma k}
$$

For repulsive electronic force, magnetic repulsive potential force $\mathrm{V}_{\mathrm{m}}$ and thermal force exerted by surrounding, in addition to the battery potential force $V_{o}$. Thus according to harmonic oscillator model

$$
\begin{gathered}
\frac{1}{2} \boldsymbol{m} \boldsymbol{v}^{2}=\boldsymbol{V}=\boldsymbol{V}_{o}+\boldsymbol{V}_{\boldsymbol{m}} \\
\boldsymbol{E}=-\gamma \boldsymbol{k} \boldsymbol{T}-\boldsymbol{p}_{\boldsymbol{m}}+2 \boldsymbol{V}_{o}+2 \boldsymbol{V}_{\boldsymbol{m}} \\
\boldsymbol{V}_{o}=\frac{\boldsymbol{E}+\boldsymbol{\gamma} \boldsymbol{k}+\boldsymbol{p}_{\boldsymbol{m}}-2 \boldsymbol{V}_{\boldsymbol{m}}}{2} \\
\boldsymbol{R}=\frac{\boldsymbol{V}_{o}}{\boldsymbol{e} \boldsymbol{I}}=\frac{\boldsymbol{E}+\gamma \boldsymbol{k} \boldsymbol{T}+\boldsymbol{p}_{\boldsymbol{m}}-2 \boldsymbol{V}_{\boldsymbol{m}}}{2 \boldsymbol{e I}}=\boldsymbol{R}_{+}+\boldsymbol{R}_{-}
\end{gathered}
$$

Then $\mathrm{R}=\mathrm{R}$.

When

$$
\boldsymbol{E}+\gamma \boldsymbol{k} \boldsymbol{T}+\boldsymbol{p}_{\boldsymbol{m}}-2 \boldsymbol{V}_{\boldsymbol{m}}<0
$$

Then

$$
T<\frac{2 V_{m}-E-p_{m}}{\gamma k}
$$

Thus the critical temperature be

$$
\boldsymbol{T}_{c}=\frac{2 \boldsymbol{V}_{m}-\boldsymbol{E}-\boldsymbol{p}_{m}}{\gamma \boldsymbol{k}}
$$

\section{Discussion}

By treating electrons as a harmonic string on the one hand [see Equations $(7,8)$ ] and by using quantum resistance on the other; a useful expression of resistance depending on V and I is found [see Eq. (10)]. Based on the assumption that the superconductor resistance is equal to the real part, the equation (14) and (15) show it vanishes below a certain critical temperature. The equation (15) proved that the critical temperature increases as mechanical pressure increases. The result of this equation agrees with some experimental studies [19]. The resulted proportional relation of critical temperature and mechanical pressure is only valid in the case of the cuprate superconductor component [23, 24]. Further, the expression (15) coincides with the conventional superconductors for low pressure and low $T_{c}$. The quantum resistance can be $\mathrm{R}$ defined in terms of energy instead of potential as equation (23) shows. The resistance here is assumed to be consisting of real Sc and imaginary part. In this case, $T_{c}$ also increases as pressure increase. The resistance vanishes below $\mathrm{T}_{\mathrm{c}}$ according to equations (23, 24, and 27). Defining $\mathrm{R}$ in terms of attractive potential [see equation (27)] or repulsive electronic force beside attractive magnetic force, [see equation (37)], and splitting $\mathrm{R}$ again to real $\mathrm{Sc}$ and negative parts, the critical temperatures $T_{c}$ [equations (33) and (40)] again depends on pressure, equation (40) shows that when pressure increase the $T_{c}$ decrease and this agrees with some superconductors compounds. Interestingly this agrees with some experimental studies $[20,25]$. However, this result is not desirable because changing material from normal state to superconductors becomes extremely difficult.

\section{Conclusion}

The plasma equation can give useful expression for energy both for the thermal and mechanical pressure. This expression, together with definition of resistance classically and quantum mechanically lead to critical temperature dependent on mechanical pressure. The uniqueness of this model is emerges fromits new approach that treats charge carriers as oscillating strings obeying plasma equation coupled with plankquantum law. It shows that the $T_{c}$ changes with pressure, in agreement with experiment. However, this model needs promotion to give an exact empirical relation between pressure and critical temperature, besides explaining other HTS phenomena and thus, could represent the standing point for further research in this regard.

\section{References}

[1] Berk, N. and Schrieffer, J. (1966) in Superconductivity. Phys. Rev. Lett, 17, 433.

[2] Poole, P. and JR. (2000) Handbook of superconductivity, A Harcourt Science and Technology Company, USA. 
[3] Christian Barth, (2013) High Temperature Superconductor Cable Concepts for Fusion Magnets, KIT scientific publishing, ISSN: 1869-1765.

[4] Charles Kittel, (2005) Introduction to solid state physics, eight editions, library of congress cataloging, USA.

[5] Sharma, R. (2015) Superconductivity Basics and Applications to Magnets, National Physical Laboratory, India.

[6] Hook, R. and Hall, E. (2010) Solid state physics, Second addition, library of congress cataloging, USA.

[7] Michel cyrot and Davor Pavuna. (1992) Introduction to superconductivity and high- $T_{c}$ materials, world scientific, Singapore.

[8] Giuseppe Grosso and Giuseppe pastori parravcini. (2003) Solid state physics, second printing, British Library Cataloguing, UK.

[9] Gupta, C. (2009) Solid state physics second revised enlarged edition, Vikas Publishing House (P) Ltd. UBS Publisher's Distributors, New Delhi.

[10] Cardwell, A. and Ginley, S. (2003) Handbook of superconducting materials volume 1: superconductivity, materials and processes, Institute of Physics Publishing, UK.

[11] Neeraj Mehta. (2009) Textbook of engineering physics part II, PHI learning private Limited, New Delhi.

[12] Uchida. (2015) High temperature superconductivity, the road to higher critical temperature, DOI 10.1007/978-4-431-553007.

[13] Wesche. (2015) Physical properties of high-temperature superconductors, John Wiley \& Sons, United Kingdom.

[14] Alexandrov, S. (2003) Theory of Superconductivity from Weak to Strong Coupling, IOP Publishing Ltd, Bristol and Philadelphia.

[15] Sin, A., Odier, P., Regueiro, M., Ordando, M., and Cunha, A. (1999) pressure effects in $\mathrm{Hg}_{0.82} \mathrm{Re}_{0.18} \mathrm{Ba}_{(2-\gamma)} \mathrm{Sr}_{y} \mathrm{Ca}_{2} \mathrm{Cu}_{3} \mathrm{O}_{8+\sigma}$, in Xavier Obradors, F. Sandiumenge, J. Fontcuberta (eds.),
Applied Superconductivity 1999, volume 1. Large scale applications, proceedings of EUCAS, the fourth European conference on applied superconductivity, held in Sitges, Spain.

[16] Narlikar, A. (2005) Frontiers in superconducting materials, Springer, Germany.

[17] Mourachkine. (2004) Room-temperature superconductivity, cambridge international science publishing, UK.

[18] Angilella and Pucci. (2000) Pressure effect in high- $T_{c}$ super conductors whith $\mathrm{n}$ inequivalent layers.

[19] Jover, D., Wilhelm, H., and Wijngaarden, R. (1997) Pressure dependence of the superconducting critical temperature of the $\mathrm{Tl}_{0.5} \mathrm{~Pb}_{0.5} \mathrm{Sr}_{2} \mathrm{Ca}_{1-\mathrm{x}} \mathrm{Y}_{\mathrm{x}} \mathrm{Cu}_{2} \mathrm{O}_{7}$ system, Physical review b, volume 55 .

[20] Alekseevsk, N., Dobrovol'sk, N., Nizhankovsk, V., and Tsebro, V. (1975) Effect of pressure on the properties of molybdenum sulfidesin the superconducting and normal states, Institute of Physical Problems, USSR Academy of Sciences, Zh. Eksp. Teor. Fiz. 69, 662-665.

[21] Altambori, A., Zakaria, A., Dirar, M., ELhussien, A., AbdALgani, R., and Abdalla, A. (2016) Quantum relation between superconductivity resistance and energy gap, global journal of engineering science and researches.

[22] Zakaria, A., Altambori, A., Dirar, M., ELhussien, A., AbdALgani, R., and Abdalla, A. (2016) Quantum effect of magnetic field in destroying superconductivity, international journal of engineering sciences \& management.

[23] Nikolay Plakida. (2013) High-temperature cuprate superconductors, experiment, theory, and applications, DOI 10.1007/978-3642-12633-8.

[24] Jover, D., Wilhelm, H., and Wijngaarden, R. (1996) Pressure dependence of the superconducting critical temperature of the $\mathrm{T}_{10.5} \mathrm{~Pb}_{0.5} \mathrm{Sr}_{2} \mathrm{Ca1}_{2 x} \mathrm{Y}_{x} \mathrm{Cu}_{2.7}$ system, physical review, volume 55.

[25] Parinov, I. (2007) Microstructure and Properties of HighTemperature Superconductors, Springer Berlin Heidelberg New York. 